\title{
SÍNDROME DE STURGE-WEBER: RELATO DE CASO DOS ACHADOS DA AVALIAÇÃO FONOAUDIOLÓGICA
}

\author{
Sturge-Weber syndrome: a case report on the results \\ of the phonoaudiological evaluation
}

\author{
Sara Virgínia Paiva Santos ${ }^{(1)}$, Laura Giotto Cavalheiro ${ }^{(2)}$
}

\begin{abstract}
RESUMO
Tema: avaliação fonoaudiológica em síndrome de rara ocorrência. Procedimentos: descrever o desempenho apresentado na avaliação fonoaudiológica clínica de uma paciente com diagnóstico genético de Síndrome de Sturge-Weber. Para isso, utilizou-se de avaliação da motricidade e funções orofaciais, das habilidades pragmática e semântica da linguagem oral, processos perceptuais visual e auditivo, funções cognitivas e aplicação do Denver II. Resultados: na avaliação da motricidade orofacial a paciente apresentou anomalias de estruturas ósseas da face, inadequação quanto à morfologia, mobilidade e tônus de todos os órgãos fonoarticulatórios, presença de reflexos de procura e sucção primitivos. Na avaliação das habilidades pragmática e semântica da linguagem oral, processos perceptuais visual e auditivo, funções cognitivas, a paciente apresentou exploração sensoriomotora, com pouca interação e atenção compartilhada e dificuldade de contato de olhos; comunicação predominantemente gestual, não havendo registros de atos comunicativos verbais. Apresentou funções comunicativas de pedido de ação, exclamativa, exploratória e protesto, e grande uso de função não focalizada. A compreensão oral mostrou-se alterada, gestos representativos esporádicos, processos perceptuais funcionais e lateralidade indefinida. No Denver II, falhou nas áreas pessoal-social, linguagem, motor fino e grosseiro. Conclusão: a paciente apresentou diagnóstico fonoaudiológico de Distúrbio de Linguagem e Disfagia Neurogênica Orofaríngea moderada sendo necessária intervenção fonoaudiológica a fim de maximizar a comunicação, bem como adequar as estruturas e funções motoras orofaciais.
\end{abstract}

DESCRITORES: Síndrome de Sturge-Weber; Hemangioma; Angiomatose

\section{INTRODUÇÃO}

Foi na última metade do século XIX que as manifestações que hoje conhecemos como Síndrome de Sturge-Weber (SSW) foram descritas e definidas clinicamente, pois nos tempos antigos acreditavase que os déficits neurológicos e os sinais da patologia representavam maldição ou estavam ligados à religiosidade ${ }^{1}$.

A SSW recebeu esse nome em decorrência da descrição de casos clínicos realizada pelos médi-

(1) Aluna do Curso de Graduação em Fonoaudiologia do Centro Universitário Jorge Amado, UNIJORGE, Salvador, BA, Brasil.

(2) Fonoaudióloga; Docente do Curso de Graduação em Fonoaudiologia do Centro Universitário Jorge Amado, UNIJORGE, Salvador, BA, Brasil; Mestre em Distúrbios da Comunicação Humana pela Universidade Federal de Santa Maria.

Conflito de interesses: inexistente cos Sturge, em 1879, e Weber, em $1922^{2}$, embora outros médicos e pesquisadores tenham acrescentado muito para a compreensão dessa síndrome, a exemplo de Doutor Schimer, que já em 1860 havia descrito o primeiro relato sobre angiomatose facial ${ }^{1}$.

Também denominada angiomatose encefalotrigeminal ${ }^{1-5}$, a SSW apresenta-se como uma alteração vascular das meninges e córtex cerebral ${ }^{2,6-8}$, de difícil ocorrência ${ }^{2,3,7-11}$ e calcula-se que a síndrome completa ocorre na população em geral à razão de 1:230.000 ${ }^{12}$. Não hereditária e congênita ${ }^{2,10,11}$, é de etiologia desconhecida, porém desconfia-se da persistência de um plexo vascular ao redor da porção cefálica do tubo neural ${ }^{5,4,8,13-18}$ e apresentase com a mesma frequência em ambos os sexos ${ }^{1}$. $O$ desconhecimento da patogênese precisa impulsiona várias revisões na literatura e estudos de 
caso sobre genética, embriogênese e a própria patologia ${ }^{8,19,20}$.

É comum aos casos de SSW o angioma cutâneo facial do tipo plano, com nervo de coloração vinhodo-Porto 7,14,21, ou "nevus flamejante", e na região de leptomeninges ${ }^{17,18,19,22}$, maxilares, tecidos moles ${ }^{2}$, podendo envolver pescoço, couro cabeludo ${ }^{14}$, tronco e membros ${ }^{8}$. Essa mancha geralmente é unilateral, podendo ser bilateral ${ }^{5,12,13,23}$, ou ausente - essa última numa proporção bem pequena ${ }^{24} \mathrm{e}$ acompanha o segmento do nervo trigêmio, sendo que apenas $10 \%$ dos portadores dessa mancha na área inervada pelo ramo oftálmico apresentam a síndrome. As manchas vinho-do-Porto em outras áreas não se associam com a síndrome de SturgeWeber ${ }^{23}$, mas a diversidade de características clínicas varia muito ${ }^{1,8}$, cada caso de Sturge-Weber é único e exibe achados em diferentes graus ${ }^{5}$.

De fato, há descrito na literatura análise de um subgrupo de pacientes com SSW com o achado raro de oclusão venosa profunda, no qual cinco dos seis casos relatados anteriormente tinham evidências de angiomatose leptomeníngea, e metade teve hemiatrofia cerebral, porém, um caso, inusitado, não apresentou angiomatose leptomeníngea, mostrando que, embora a avaliação inicial possa apresentar-se normal, os pacientes podem apresentar mais tarde as características clínicas da SSW ${ }^{17}$.

Os principais sinais da SSW são a angiomatose da leptomeninge homolateral ${ }^{11,17,19,25}$ e nervo facial em território do trigêmio ${ }^{1,5,8}$, com manifestações clínicas de lesões cutâneas ${ }^{10,11}$ de localização superficial ${ }^{26}$. Da gama de manifestações pode-se encontrar alteração gengival como hiperplasia gengival ${ }^{2,3} \mathrm{e}$ hemangiomas intrabucais ${ }^{2} \mathrm{e}$ de vias aéreas superiores ${ }^{26}$; alteração do sistema estomatognático ${ }^{20}$, deficiência mental ${ }^{24}$, glaucomas e demais afecções oculares ${ }^{8,11,15,21,23,24}$, macrocefalia, hemiplegia espástica, paresias e hemiparesias ${ }^{17}$, cefaleias frequentes ${ }^{23}$, atraso do desenvolvimento motor ${ }^{3,13}$, déficits neurológicos focais ${ }^{7}$, assimetria craniana ${ }^{12}$ e facial ${ }^{23}$, crises convulsivas $7,9,15,17,19$ e epilepsia ${ }^{10,18,22,24}$, do tipo Focal como um sinal comum e, em alguns casos, têm sido relatadas convulsões generalizadas ${ }^{25}$.

A literatura mostrou relato de caso de associação da SSW com a síndrome Klippel-Trenaunay, também de rara ocorrência, o que evidencia a possível associação da SSW com demais afecções ${ }^{15}$. Estudos mostram que não há uma clara distinção entre SSW e síndrome Klippel-Trenaunay e afirmam existir uma sobreposição clínica e biológica, o que exibe uma complexidade de tais fenótipos, de fato, o paciente em estudo preenchia os critérios diagnósticos de ambas as síndromes ${ }^{18}$.
Hipotireoidismo central é outro diagnóstico associado à SSW. Sabe-se que indivíduos com SSW apresentam maior prevalência de deficiência hormonal que indivíduos normais, uma vez que a medicação anticonvulsivante pode levar a anormalidades em teste de função tireoidiana e ser risco adicional de desenvolvimento da disfunção hipotálamo-hipófise secundária à disfunção do Sistema Nervoso Central ${ }^{7}$.

A SSW pode ser classificada em tipos I, II e III. O tipo I é a forma clássica da síndrome, caracterizada pelos angiomas faciais e leptomeníngeos na presença de glaucoma. O tipo II, no entanto, é diferenciado pela inexistência de associação com doença intracraniana, e o tipo III com glaucoma, sendo que nesta, o angioma apresenta-se de forma isolada ${ }^{27}$.

A alteração displásica vascular que caracteriza a SSW ocorre provavelmente da $5^{\mathrm{a}}$ a $8^{\mathrm{a}}$ semana após a concepção ${ }^{8,14}$. Já as crises epilépticas, a hemiplegia espástica e a deficiência mental se manifestam mais frequentemente no primeiro ano de vida ${ }^{12}$, podendo existir diferenciações.

Casos descritos na literatura ajudam a entender o desempenho de linguagem, cognitivo, motor e orofacial da SSW, conforme as descrições abaixo:

Estudo comparativo retrospectivo realizado com 102 pacientes com SSW, para determinação da diferença do prognóstico entre pacientes que tinham comprometimento uni e bilateral dos hemisférios cerebrais, mostrou que todos apresentaram alterações de inteligência com maior comprometimento para os casos de comprometimento bilateral (45\% do lesados unilaterais apresentaram inteligência média para apenas $8 \%$ dos lesados bilateralmente). $O$ envolvimento bilateral do cérebro por SSW, se comparado com o envolvimento unilateral, parece estar associado a convulsões precedentes, o que ocasiona um pior prognóstico para o desenvolvimento mental ${ }^{28 .}$

Relato de caso de uma paciente que falou e andou em torno de um ano de idade, nascida de parto normal, a termo, sem intercorrências, com choro ao nascimento, sem antecedentes de outras doenças ou traumatismos cranianos. As crises convulsivas constantes apareceram aos cinco meses de idade, mas foram controladas com uso de Fenobarbital. Os exames físico intrabucal, neurológico e oftalmológico evidenciaram hiperplasia gengival, incompatibilidade para a idade e glaucoma ipsilateral ao hemangioma, respectivamente ${ }^{5}$.

Em outros relatos ${ }^{3}$ observou-se presença de crises convulsivas nos dois casos descritos, sendo que no primeiro caso as crises se manifestavam há mais de 20 anos e controladas com medicação somente nos últimos 3 anos; cursou com leve déficit de inteligência. Já no segundo caso, o paciente era 
portador de Paralisia Cerebral congênita além da SSW, e as crises estavam presentes desde o nascimento com aumento de ocorrência diária no último ano, mesmo com terapêutica medicamentosa anticonvulsivante e cursou com atraso grave no desenvolvimento mental. No exame intrabucal observaram hiperplasia gengival em ambos os casos.

Um quarto caso ${ }^{9}$, que merece ser relatado, diz respeito a um paciente nascido de parto normal, que apresentou febre, crises convulsivas e diarreia aos 3 meses de idade, só voltando a apresentar novos episódios de crises, que foram aumentando a frequência, chegando a 1-2 casos diários, aos 3 anos de idade, quando até esse período apresentava aleitamento e desenvolvimento normais. A história traz dados relevantes como início da fala e a deambular com um ano de idade, pouca sociabilidade e agressividade, e progressividade das alterações oculares e dentárias.

Outro relato de caso, referente a um neonato, nascido de 39 semanas gestacionais, sexo masculino, de cariótipo normal, com caracterítica mancha vinho do porto envolvendo couro cabeludo frontal e na parte interna da pálpebra, assimetrias intracranianas cursando com hemiplegia de membro superior, hemianopsia e, apesar de grave anomalia cortical não houve aparecimento de convulsões ou características epilépticas durante dois anos de seguimento. Apresentou assimetria de pálpebra e de estruturas ósseas e foi capaz de utilizar cerca de 10 palavras, virar as páginas de uma revista e indicar imagens com o seu dedo indicador, esses últimos dados importantes para melhor caracterização dos casos de SSW, devendo ser melhor descritos ${ }^{14}$.

Outro estudo descreveu o desenvolvimento de uma criança acometida pela SSW, que comprometia o hemisfério esquerdo com fortes convulsões. A compreensão de palavras e comandos simples permaneceu estagnada por 48 meses, aproximadamente, com dificuldade persistente para o desenvolvimento do discurso. Entretanto, após hemisferectomia esquerda e retirada de anticonvulsivantes observou-se, por volta dos nove anos de idade, progresso bastante significante na linguagem e na fala, em aspectos semânticos, prosódicos, gramaticais e fonológicos, bem como das demais funções cognitivas, alcançando pontuações significativas em testes de linguagem expressiva e receptiva, o que não o distancia ou o coloca em desvantagem em relação aos resultados alcançados por crianças que também realizaram retirada do hemisfério esquerdo e que já apresentavam, inclusive, início do desenvolvimento da fala e da linguagem ${ }^{16}$.

A literatura mostrou ainda paciente acometido pela SSW, na qual foi realizada lobectomia temporal anterior devido epilepsia intratável apresentando crises parciais complexas após os oito meses de nascimento e, posteriormente, graves mudanças de temperamento, podendo essas estar relacionadas a uma alteração nas amígdalas. Ambas as afecções foram tratadas com sucesso com a retirada do lobo temporal anterior ${ }^{22}$.

Segundo relato, 39\% dos casos apresenta inteligência normal ${ }^{13}$. Outro estudo revisou as características clínicas de pacientes acometidos pela SSW visto durante um período de 40 anos e mostrou que apenas $36 \%$ dos estudados apresentaram baixa inteligência ou limítrofe, não havendo relação entre o tamanho do nevo facial e a gravidade da lesão cerebral ${ }^{24}$.

A limitação intelectual e a baixa sociabilidade, associadas à má aparência estética dificultam a integração social do indivíduo com SSW ${ }^{24}$, podendo este aspecto servir de incentivo para novas pesquisas na área.

Há, ainda, descrição na literatura de diagnóstico de SSW sem sintomas neurológicos, embora na presença de calcificações cerebrais. O paciente de 11 anos, sexo masculino, apresentando grave glaucoma bilateral associado a nevo facial também bilateral, põe em questão as peculiaridades dessa síndrome, fomentando crescimento de estudos epidemiológicos, clínicos e do prognóstico, já que se apresenta como uma variante das apresentações clássicas ${ }^{11}$.

Outro relato de caso confirma a inexistência de alteração neurológica temporária, de paciente assintomático até os 24 anos de idade, quando se queixou de enxaqueca, déficit visual e hemiparesia. Após 10 anos, no entanto, esse paciente desenvolveu grave epilepsia refratária e enxaqueca, que poderia durar várias horas - como mostrado no eletroencefalograma - o que pode ter levado a progressiva deterioração neuronal ${ }^{10}$.

A terapia envolve administração de fármacos antiepilépticos, que possuem um valor limitado podendo causar hiperplasia gengival; medidas cirúrgicas podem ser necessárias para controle das convulsões ${ }^{10,13}$, somente em casos de lesão extensa ${ }^{12}$, porém se faz necessário uma avaliação criteriosa quanto ao planejamento cirúrgico. A laserterapia é uma opção para melhoria do nevo facial vinho do porto ${ }^{5} \mathrm{e}$ é recentemente indicada como a melhor abordagem, mas não como a mais satisfatória ${ }^{21}$. A reabilitação oral de pacientes com a SSW é normalmente complexa, e requer procedimentos conservadores ${ }^{12}$.

Estudos têm sido desenvolvidos com ênfase nos aspectos fisiopatológicos, no tratamento, na intervenção ${ }^{10,26}$ e, sobretudo, na busca da prevenção da lesão cerebral presente na SSW por 
meio de exames de imagem ${ }^{19}$ que, atualmente, mostram-se úteis para o diagnóstico precoce ${ }^{10,23,29}$. É sabido que recentemente, um estudo revelou que a Ressonância Magnética com DTI (Diffusio Tensor Imaging) pode ser um método clinicamente útil para avaliação da integridade do trato cortiespinal em crianças pequenas que apresentam risco para disfunção motora progressiva ${ }^{30}$. Esse, no entanto, representa apenas um passo de um longo caminho a ser percorrido por meio de investigações sempre mais detalhadas para o favorecimento dos portadores da SSW ${ }^{31}$.

Em vista da variedade de alterações encontradas na SSW é de fundamental importância a avaliação periódica e acompanhamento dos casos por parte de uma equipe interdisciplinar que envolva também fisioterapeutas, terapeutas ocupacionais e psicólogos ${ }^{20,23}$, sobretudo pela indefinição etiológica, e não somente isso, mas de profissionais que estejam abertos à discussão e estudo dos casos para maior conhecimento das diversas manifestações possíveis, das terapêuticas adotadas em cada caso, de melhor delimitação do prognóstico e expectativa de melhor qualidade de vida desses pacientes.

A escassez de estudos relatando manifestações fonoaudiológicas na SSW, associada à raridade de ocorrência, justifica o nosso interesse em realizar tal estudo, para colaboração de maior conhecimento por parte dos fonoaudiólogos e profissionais de saúde, e útil enquanto incentivo para mais pesquisas nesse campo pouco explorado.

Em janeiro de 2008 foi atendida uma paciente com diagnóstico genético de SSW, no Setor de Fonoaudiologia do Centro Universitário Jorge Amado - UNIJORGE/BA, situado em Salvador, sendo, pois, o objetivo desse estudo descrever 0 desempenho apresentado por essa paciente na Avaliação Fonoaudiológica Clínica.

\section{APRESENTAÇÃO DO CASO}

\section{Anamnese}

A paciente foi submetida à Anamnese Fonoaudiológica sendo possível caracterizar a queixa e buscar os possíveis fatores etiológicos; abordar dados desde o período gestacional e condições de nascimento e desenvolvimento motor geral, orofacial, de linguagem, auditivo, cognitivo, social e escolar; delimitar o estado geral de saúde da paciente, uso de medicamentos e atendimento ou acompanhamento com outros profissionais de saúde, bem como informações complementares acrescentadas pelos informantes, os pais da criança.

A paciente em estudo, com três anos e nove meses de idade, não-estudante, apresentava diagnóstico médico de SSW há 2 anos. A principal queixa trazida pelos pais foi a ausência de oralidade. Nascida de 32 semanas, de gestação gemelar e raça branca, a paciente começou a apresentar febre aos 7 meses de nascida e crises convulsivas tônicas de difícil controle a partir dos 12 meses de idade - que permaneceram até o momento, mesmo com a administração de terapia anticonvulsivante quando apresentava apenas poucas vocalizações sem sentido contextual, choro usualmente e inabilidade para expressar seus sentimentos. Os informantes acrescentaram ainda que a criança apresenta desequilíbrios ao deambular, inabilidade de controle esfinctérico, engasgos esporádicos à alimentação, ausência de dificuldades respiratórias, não demonstra interesse por jogos ou brincadeiras, não focalização da atenção nem demonstrações de aprendizado de situações habituais.

Os informantes relataram não ter havido intercorrências como hipóxia, icterícia, infecções ou transferência para unidades de terapia semi ou intensiva depois do nascimento da criança.

Após a realização da anamnese, deu-se início à avaliação fonoaudiológica clínica da Motricidade Orofacial (MO), de linguagem oral, incluindo as habilidades pragmática e semântica assim como os processos perceptuais visual e auditivo, funções cognitivas atenção, lateralidade de olhos, membros e ouvidos, conduta e aplicação do Denver II (TTDD) ${ }^{32}$. As demais habilidades da linguagem oral como sintaxe e fonologia, assim como a fala, a fluência, a articulação, a voz, o raciocínio lógicomatemático e a linguagem escrita não foram avaliados devido à ausência de oralidade e/ou falta de colaboração por parte da paciente.

Para a avaliação da Motricidade Orofacial (MO) foi utilizado o Protocolo para Exame Clínico de Avaliação das Funções Miofuncionais Orofaciais de Marchesan (2005) ${ }^{33}$. A avaliação da MO foi realizada por meio de avaliação miofuncional passiva, devido à falta de compreensão e colaboração da paciente. A avaliação dos itens B: Corpo, C: Face e D: Funções orais do Protocolo utilizado, foi realizada por meio da antroposcopia ou avaliação visual, seguidas de avaliação complementar com inspeção oral e ausculta laríngea.

As Habilidades da Linguagem Oral, Pragmática e Semântica, bem como os processos perceptuais e as funções cognitivas, foram avaliadas por meio da observação dos recursos comunicativos utilizados pela criança e do seu desempenho durante as atividades lúdicas (contextualizadas), eliciadas pela terapeuta, procedimento metodológico sugerido por Garcia, Rossi e Giacheti ${ }^{34}$. Buscou-se utilizar cores vivas e chamativas durante a avaliação fonoaudiológica clínica para atrair a atenção da paciente, 
levando em consideração as alterações visuais presentes no caso em estudo. Na avaliação da pragmática e semântica foram utilizados os parâmetros de comparação dos resultados propostos por Fernandes ${ }^{35}$.

O TTDD foi baseado na observação direta das ações realizadas pela criança e no relato dos pais. É composto pelos níveis Pessoal-Social, Motor Fino-Adaptativo, Linguagem e Motor Grosso, e pelos itens: avançado, normal, cautela e atraso. Este teste foi escolhido devido a sua grande aplicabilidade, sobretudo em estudos brasileiros ${ }^{36}$.

Os itens para determinação dos resultados do TTDD baseiam-se na possibilidade da criança acertar, falhar ou recusar nos itens desenvolvidos como esperado para a maioria das crianças da amostragem de padronização.

O Denver II é usado para identificar a criança cujo desenvolvimento parece estar atrasado em comparação com o desenvolvimento de outra criança, ou seja, leva em consideração o avanço da idade, no entanto o teste não faz diagnóstico, mas alerta quanto à presença de deficiências e indica a necessidade de investigação mais aprofundada. A interpretação do teste, como manda o Manual ${ }^{37}$ é feita da seguinte maneira:

- normal: nenhum atraso e no máximo 1 cautela;

- anormal: 2 ou mais atrasos;

- suspeito: 1 atraso e 2 ou mais cautelas.

- não-testável: quando a criança se recusa a execução de itens que impedem a apreciação de seu desempenho em uma ou mais áreas.

O diagnóstico não-testável foi desconsiderado por não avaliar o desempenho da paciente, não contribuindo, dessa forma, para confirmação ou não dos achados da avaliação clínica ${ }^{27}$.

Foi assinado Termo de Consentimento Livre Esclarecido pelos responsáveis da paciente, segundo resolução no 196/96. A presente pesquisa passou pela Comissão de Ética do Centro Universitário Jorge Amado - UNIJORGE/BA, com protocolo de número 0001/2007. A paciente foi encaminhada para terapia fonoaudiológica.

\section{RESULTADOS}

A avaliação fonoaudiológica realizada foi didaticamente dividida em subitens para melhor visualização dos resultados obtidos.

De acordo com o protocolo seguido observou-se:

\section{Antroposcopia}

Item B - I, II, III e IV (Corpo): inclinação de cabeça em relação ao corpo, rotação anterior de ombros, cabeça anteriorizada em relação ao tórax e inclinada para frente.
Item C - I até XII (Face): assimetria de olhos, nariz, orelhas, lábios, bochechas e músculo mentual, lábios entreabertos, desvio de comissura labial, rigidez de lábios, bochechas e músculo mentual de possível discrepância maxilo-mandibular - bem como restrição da mobilidade e desvio de mandíbula, posição habitual de língua anteriorizada.

Item D - I e III (Funções orais): observou-se durante todo o exame respiração predominantemente oronasal e saída de ar diferenciada no teste com espelho de Glatzel, deglutição com movimentação de cabeça e contração da musculatura periorbicular.

\section{Inspeção Oral}

Item C - VIII até XI (Face): assimetria de língua e de palato duro e de palato mole, desvio de úvula, dentição decídua, presença de diastemas, alteração gengival, desvio de linha média dentária e óssea.

A paciente apresenta nevo cutâneo de face unilateral à direita, inadequação postural e escape extra-oral de saliva constante. Observou-se, ainda, má-oclusão dentária e alterações de sensibilidade. A paciente apresentou reflexos de procura e sucção primitivos, que não impediram o desenvolvimento dos padrões de deglutição e alimentação.

Tais alterações revelam anomalias de estruturas ósseas da face, inadequação quanto à morfologia, mobilidade e tônus dos órgãos fonoarticulatórios (OFAs), assimetria facial e alteração de sensibilidade.

As provas sugeridas pelo protocolo para Exame Clínico de Avaliação das Funções Miofuncionais Orofaciais de Marchesan ${ }^{33}$ não foram realizadas uma vez que exigem a participação do paciente. A avaliação oromiofuncional foi realizada de forma passiva.

Testou-se a consistência líquida com oferecimento de água mineral natural. As consistências de líquido engrossado, pastoso, pastoso fino e sólido não foram testadas devido a não aceitação e colaboração por parte da paciente.

Observou-se escape extra-oral devido ao volume excessivo ingerido pela paciente e, concomitantemente, tosse durante e após a deglutição para a consistência oferecida.

\section{Avaliação complementar: ausculta laríngea}

$\mathrm{Na}$ ausculta laríngea foi possível identificar sinais clínicos de penetração no nível das pregas vocais, respiração ruidosa seguida de desconforto respiratório após a deglutição da consistência líquida apenas, uma vez que as demais consistências não foram testadas. 


\section{Habilidades Pragmática e Semântica da Linguagem Oral}

A comunicação da paciente revelou-se predominantemente gestual (Figura 1), não havendo registros de atos comunicativos verbais $(0 \%)$, relativa utilização do meio comunicativo vocal (10\%), e predominância do meio comunicativo gestual $(90 \%)$. Foi possível identificar funções comunicativas de pedido de ação, exclamativa, exploratória e protesto, com grande uso de função não focalizada. Observou-se iniciativa dialógica prejudicada, intenção e/ou desejo de comunicar-se, o que não foi diferente para averiguação do tempo atencional que se mostrou reduzido.

A compreensão oral mostrou-se alterada na testagem para ordens simples e comandos complexos. A observação do comportamento da criança não evidenciou uso de expressões com significado relacional e contextual funcionais, com gestos representativos predominantemente esporádicos.

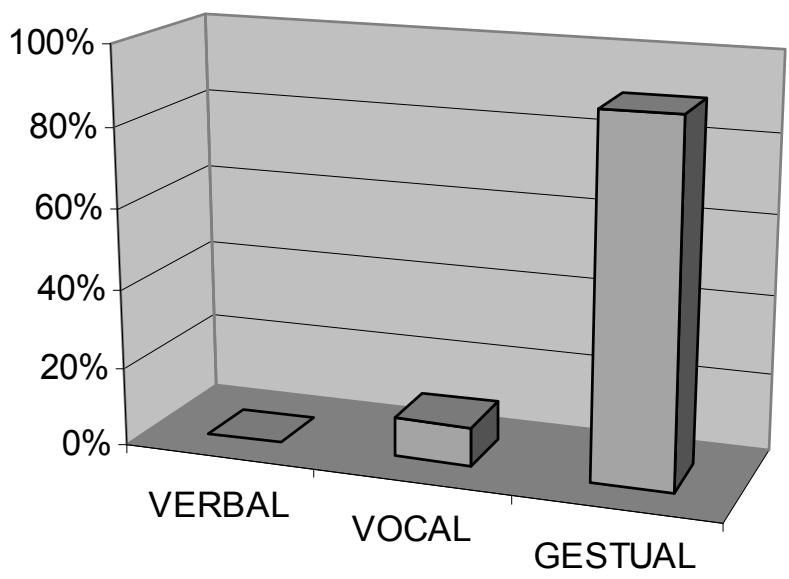

Figura 1 - Utilização dos meios comunicativos pela criança

\section{Processos Perceptuais e Funções Cognitivas}

$A$ avaliação dos processos perceptuais, visual e auditivo, para realização de percepção, discriminação, análise-síntese e figura-fundo, auditivas e visuais, mostrou-se funcional. A lateralidade de membros, olhos e ouvido mostrou-se indefinida, uma vez que não foi observada predominância direita ou esquerda.

A paciente não demonstrou alterações de conduta como agressividade ou constante inquietude mesmo frente a situações diversificadas.

A ausência de contato de olhos no momento da avaliação - não desconsiderando, claramente, a alteração visual existente -, associada à dificuldade semântica e cognitiva, evidenciou o desinteresse da paciente pelo interlocutor o que não permitiu estabelecimento de troca de turno ou mesmo manutenção dos temas propostos por parte da paciente, uma vez que houve predominância de exploração sensório-motora com pouca interação e atenção compartilhada.

\section{Denver II}

Os resultados do Denver II demonstraram atraso nos quatro níveis quando comparados ao desenvolvimento esperado para a idade cronológica da paciente (Figura 2). O nível Pessoal-Social revelou apenas $4 \%$ de presença das ações abordadas e esperadas para a idade cronológica da paciente, aparecendo em seguida o nível Motor Fino com $8,33 \%$, Linguagem $14,28 \%$ e Motor Grosso de $59,26 \%$.

\section{DISCUSSÃO}

A paciente em estudo é portadora da forma clássica da SSW, tipo I, com angioma cutâneo facial unilateral acompanhando o segmento do nervo

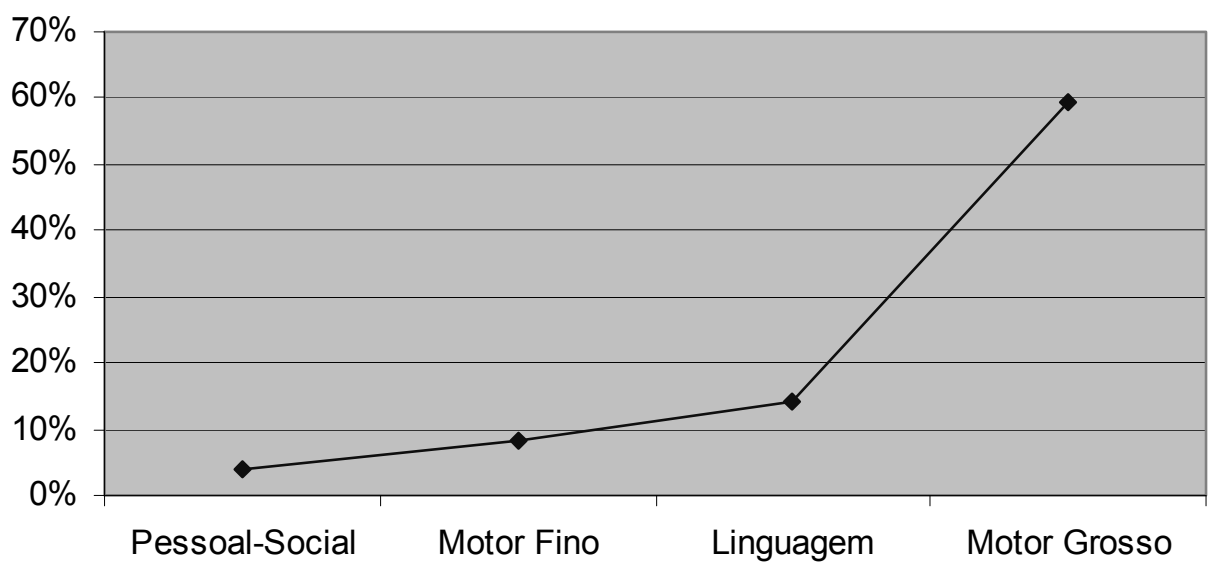

Figura 2 - Resultados dos níveis que compõem o Denver II 
trigêmio, e glaucoma ipsilateral ao hemangioma. Apresentou dados de anamnese e achados da avaliação fonoaudiológica compatíveis com a literatura, respectivamente:

- presença de crises convulsivas após o nascimento, diagnóstico médico de Deficiência Mental e reduzida sociabilidade ${ }^{3,9,13}$.

- hiperplasia gengival ${ }^{2,3}$, afecções morfofuncionais faciais e intrabucais e, assim como os resultados da antroposcopia de alteração de estruturas ósseas, má-oclusão e assimetria facial 12,14,23.

A convulsão é a desordem neurológica mais comumente observada ${ }^{4}$, com maior predominância para pacientes com envolvimento bilateral (93\%) que unilateral $(72 \%)$, ocorrendo em $56 \%$ dos casos ${ }^{13}$. O aparecimento das crises convulsivas de difícil controle pode ser atribuído à obstrução do retorno venoso, ocasionado pela malformação vascular leptomeníngea, característica da síndrome. Pode-se inferir que a obstrução do retorno sanguíneo produziu anormalidade na drenagem nos plexos profundos e hipertrofia do plexo coroide, como referido na literatura ${ }^{6}$, que observou associação de ausência de convulsões quando na existência de maior fluxo sanguíneo cerebral.

A disfunção neurológica da paciente revela o retardo do desenvolvimento motor como um achado precoce. De fato, os resultados dos níveis motor fino-adaptativo e motor grosso do Denver II mostraram-se alterados, compatíveis com a queixa trazida pelos responsáveis da paciente de dificuldade na deambulação, desequilíbrio, e ausência de controle de esfíncteres. Há, no entanto, discordância na literatura que traz dados de uma paciente que deambulou com um ano de idade ${ }^{9}$. De fato, cada caso pode exibir achados diferenciados ${ }^{5}$ considerando a grande diversidade humana.

Foi possível identificar na revisão da literatura que a alteração cognitiva não é determinante, sendo $39 \%$ dos casos com inteligência normal ${ }^{12,13}$. Já em 1992 pesquisadores haviam descrito a existência de casos leves, sem comprometimento das faculdades mentais ${ }^{12}$, diferentemente do caso em estudo.

As alterações estruturais orofaciais, apesar de permitirem a execução das funções orais, as permitem de maneira inadequada, o que gera um conjunto de comprometimentos nos mecanismos necessários para uma mastigação, deglutição, respiração e, provavelmente fonação, eficientes.

A deglutição é um processo sinérgico composto por fases intrinsecamente relacionadas, sequenciais e harmônicas, e a sua eficiência exige complexa ação neuromuscular, integridade das vias aferentes, integração dos estímulos no sistema nervoso central, vias eferentes, resposta motora, integridade das estruturas envolvidas e comando voluntário. Tudo isso precisa estar em comunhão com preservação de sensibilidade, paladar, propriocepção, mobilidade, tônus e tensão muscular, além da intenção de se alimentar ${ }^{38}$.

Como observado nos resultados descritos, há presença de alterações na sensibilidade, mobilidade e tônus, ou seja, persiste um déficit em pelo menos um dos pré-requisitos para uma deglutição eficiente: a integridade das estruturas orais envolvidas. No caso da paciente em estudo, o posicionamento inadequado de língua, associado às alterações morfológicas e funcionais dos OFAs, associada à inabilidade do controle de esfíncter laríngeo, caracterizam o quadro de disfagia, pondo em risco, consequentemente, proteção de vias aéreas superiores uma vez que não se consegue o mister sinergismo entre elevação e anteriorização da laringe e fechamento glótico, com adequada pressurização, deslocamento da epiglote sobre o ádito laríngeo e apneia protetora ${ }^{39}$.

A disfagia é classificada como orofaríngea neurogênica moderada, uma vez que se identifica comprometimento neurológico e dificuldade no transporte oral do bolo associado a sinais sugestivos de penetração laríngea como presença de tosse durante e após a deglutição, respiração ruidosa e desconforto respiratório - provável ocorrência de estase em recessos faringeais - e pequena quantidade de material aspirado. Na ausculta laríngea foi possível identificar sinais clínicos de penetração no nível das pegras vocais, sugestivo de disfunção no trânsito do alimento para o estômago ${ }^{38}$.

Os resultados insatisfatórios da avaliação dos aspectos pragmáticos e semânticos da linguagem justificam o distúrbio de linguagem, comprometimento esse que interfere, desde já, direta e significativamente no desenvolvimento social, pessoal e afetivo dessa criança.

As funções comunicativas exploratória e não focalizada estão em destaque em relação às demais. A literatura revela que a função comunicativa mais frequentemente utilizada aos seis meses de idade é a exploratória, com diminuição de expressão de atos não focalizados para $1 \%$ a partir dos 18 meses, quando se espera que o número de funções comunicativas triplique nessa faixa etária, o que não se observou no presente caso; há ainda predominância de atos não focalizados e não se observou número de 15 funções comunicativas expressas, como esperado para faixa de normalidade ${ }^{35}$.

A comunicação está limitada a gestos e vocalizações indiferenciadas - compatível com a queixa trazida pelos informantes - como ausência de intenção comunicativa, sendo os primeiros usos 
intencionais da comunicação presentes desde os 3 meses de idade ${ }^{35}$. A paciente em estudo aos sete meses de idade ainda não apresentava intencionalidade, mas vocalizações raras e sem significado para as situações cotidianas, expressandose de forma rudimentar através do choro. Os atos comunicativos por minuto são insignificantes para a pesquisa $(0 \%)$, sendo o padrão considerado de 6 a 8 atos comunicativos por minuto para a idade cronológica da paciente ${ }^{35}$. A predominância do meio comunicativo gestual revela um padrão de desenvolvimento ascendente ou ainda em desenvolvimento, de fato, até aproximadamente o quarto mês de vida há um equilíbrio entre o uso de gestos e vocalizações, mas a partir do sexto mês os gestos passam a corresponder a dois terços de toda a comunicação expressa, inclusive até o início da comunicação verbal, em torno dos 12 meses; a partir dos 15 meses passa a haver um novo equilíbrio entre a comunicação gestual e a soma das verbalizações e vocalizações ${ }^{35}$. A paciente em estudo parece localizar-se nesse intermédio, antecedendo um momento que exige maior complexidade e organização dos comandos e organizações cerebrais.

Dessa forma, os déficits apresentados pela paciente em estudo na linguagem oral, de compreensão, impossibilitam até o presente momento, a existência de atividade conversacional, uma vez que a paciente não consegue construir significados a partir do mundo ao seu redor e atribuir funcionalidades, associar eventos e fatos, ou estabelecer relações de causalidade e efeito. As limitações de comunicação e compreensão existentes funcionam como obstáculos para a expressão eficiente e inteligível da paciente, e, sobretudo, dificultam o estabelecimento das relações interpessoais.

Os resultados do Denver II apontam interpretação de resultado anormal, uma vez que os itens testados encontram-se com atraso significante. $O$ nível motor grosso é o que apresenta maiores índices de porcentagem em detrimento de linguagem, do motor fino e pessoal-social, o que mostra a incapacidade de realização de atividades motoras finas, de tecer relações sociais e linguísticas por parte da paciente.

Todos os itens avaliados encontram-se alterados. Sendo assim, em função dos achados, o diagnóstico fonoaudiológico na SSW foi de: Distúrbio de Linguagem e Disfagia Orofaríngea Moderada.

Não foram encontrados na literatura relatos de casos, ou mesmo outros estudos, relacionando os sinais e sintomas presentes na SSW com achados de avaliação fonoaudiológica, porém a caracterização desse quadro, com suas usuais manifestações não diferiram das encontradas por outros autores.

Como pôde ser observado na descrição acima, os cinco subitens demonstraram um perfil que foge à normalidade ou ao que é esperado para a idade cronológica da paciente.

\section{CONCLUSÃO}

Conforme desempenho explicitado, a paciente apresenta diagnóstico fonoaudiológico de Distúrbio de Linguagem e Disfagia Orofaríngea Neurogênica Moderada, o que demonstrou alteração de comunicação e socialização, dos padrões de deglutição e funções orofaciais. Os achados revelaram a necessidade de terapia fonoaudiológica para a paciente em questão com SSW a fim de maximizar a comunicação com o estabelecimento e aprimoramento das relações interpessoais, bem como adequação das estruturas e funções motoras orofaciais para melhora de sua qualidade de vida.

Dessa forma, incentiva-se pesquisas relacionadas à SSW, mais especificamente em relação às manifestações fonoaudiológicas encontradas e relatos de acompanhamento terapêutico. A escassez de estudos relatando manifestações fonoaudiológicas na SSW, associada à raridade de ocorrência, impulsiona pesquisas nesse vasto campo a ser explorado. 


\section{ABSTRACT}

Background: phonoaudiological evaluation of rare occurrence syndrome. Procedures: describe the performance shown in phonoaudiological clinical evaluation of a patient with genetic diagnosis of Sturge-Weber Syndrome. Evaluation of the drive and orofacial functions, semantic and pragmatic skills of oral language, perceptual visual and auditory processes, cognitive functions and test development of Denver II. Results: in the assessment of orofacial drive the patient had abnormalities concerning bone structures of the face, inadequate as for the morphology, mobility and tone of all speech organs, presence of reflections of demand and sucking primitive. In the assessment of skills and Semantic Pragmatic of oral language and Speech, perceptual processes visual and auditory, cognitive functions the patient had sensoriomotor exploitation, with little interaction and shared attention of the patient and difficulty of eye contact; predominantly gestural communication, with no records of verbal communicative acts. The communicative functions of a request for action, exclamatory, exploratory and protest, and great use of non-focused light. Listening proved to be altered, representative gestures were sporadic, processes and perceptual functional laterality undefined. In the test development of Denver II, the patient failed in the areas: personal, social, language, motor fine and coarse. Conclusion: the patient showed speech diagnosis of phonoaudiological language and speech disturbance and moderate Oropharyngeal Neurogenic Dysphagia, being required to maximize communication, and adapt the structures and orofacial motor functions.

KEYWORDS: Sturge-Weber Syndrome; Hemangioma; Angiomatosis

\section{REFERÊNCIAS}

1. Ball K. History of Sturge-Weber syndrome. \{homepage da internet] Sturge-Weber Foundation: UK; 2007. Disponível em URL: http://www.sturgeweber.org/SWSHistory.aspx

2. Bhansali RS, Yeltiwar RK, Agrawal AA. Periodontal management of gingival enlargement associated with Sturge-Weber syndrome. J Periodontol. 2008; 79(3):549-55.

3. Pereira CM, Vedrano APG, Naclério-Homem MG. Síndrome de Sturge-Weber: revisão de literatura e relato de dois casos clínicos. Rev Bras Patol Oral. 2003; 2(1):44-8.

4. Marinho AS, Cherubini K. Síndrome de SturgeWeber. Rev Odontol Univ Cidade SP. 2006; 18(2):167-73.

5. Gomes ACA, Silva EDO, Albert DGM. Síndrome de Sturge-Weber: relato de caso clínico. Rev Cirur Traumatol Buco-Maxilo-Fac. 2004 jan-mar; 4(1):47-52.

6. Pinton F, Chiron C, Enjolras O, Motte J, Syrota A, Dulac O. Early single photon emission computed tomography in Sturge-Weber syndrome. J Neurol Neurosurg Psychiatr. 1997; 63(5):616-21.

7. Comi AM, Bellamkonda S, Ferenc LM, Cohen BA, Germain-Lee EL. Central hypothyroidism and Sturge-Weber syndrome. Pediatr Neurol. 2008; 39(1):58-62.
8. Ch'ng S, Tan ST. Facial port-wine stains - clinical stratification and risks of neuro-ocular involvement. J Plast Reconstr Aesthet Surg. 2008; 61(8):889-93. 9. Mesquita AP, Muffarrej W, Monteiro PAC. Síndrome de Sturge-Weber. Caso clínico. Brasil Dermat. 1975; 50:165.

10. Jacobs J, Levan P, Olivier A, Andermann F, Dubeau F. Late-onset epilepsy in a surgicallytreated Sturge-Weber patient. Epileptic Disord. 2008; 10(4):312-8.

11. Ouaggag B, Baha Ali T, Gaboune L, Benfdil $\mathrm{N}$, Moutaouakil A. [Bilateral Sturge-Weber-Krabbe syndrome. A case report]. Bull Soc Belge Ophtalmol. 2008; (307):19-23.

12. Wiedemann R. Síndrome de Sturge-Weber. In: Kunze J, Dibbern H. Atlas de síndromes dismórficas. São Paulo: Manole; 1992. p. 356 -7.

13. Hamartoses P. Sequência de Sturge-Weber. In: Kenneth LJ. Smith padrões reconhecíveis malformações congênita. São Paulo: Manole; 1998. p. 495.

14. Portilla $P$, Husson $B$, Lasjaunias $P$, Landrieu $P$. Sturge-Weber disease with repercussion on the prenatal development of the cerebral hemisphere. Am J Neuroradiol. 2002; 23(3):490-2.

15. Rahman M, Rahman S, Rahman M, Akhter S, Kawser C. Overlapping of Sturge Weber syndrome and Klippel Trenaunay Weber syndrome. Mymensingh Med J. 2008 Jan; 17(1):78-81.

16. Vargha-Khadem $F$, Carr LJ, Isaacs E, Brett E, Adams C, Mishkin M. Onset of speech after left 
hemispherectomy in a nine-year-old boy. Brain. 1997; 120(Pt1):159-82.

17. Slasky SE, Shinnar S, Bello JA. Sturge-Weber syndrome: deep venous occlusion and the radiologic spectrum. Pediatr Neurol. 2006; 35(5):343-7.

18. Rafai MA, Otmani HE, Boulaajaj FZ, Sibai M, Moutaouakkil F, Chlihi A, et al. [Sturge--WeberKlippel--Trenaunay syndrome (case report)]. J Mal Vasc. 2008; 33(1):35-8.

19. Baselga E. Sturge-Weber syndrome. Semin Cutan Med Surg. 2004; 23(2):87-98.

20. Santos SVP, Cavalheiro LG. Avaliação fonoaudiológica na síndrome de Sturge-Weber: um estudo de caso. Pró-Fono. 2008 20(Supl):26-8.

21. Hennedige AA, Quaba AA, Al-Nakib K. SturgeWeber syndrome and dermatomal facial port-wine stains: incidence, association with glaucoma, and pulsed tunable dye laser treatment effectiveness. Plast Reconstr Surg. 2008; 121(4):1173-80.

22. Utsunomiya $T$, Shimizu $H$, Sunaga $S$, Sugano $\mathrm{H}$, Arai $\mathrm{N}$. [A case of Sturge-Weber syndrome with severe temper tantrum]. No Shinkei Geka. Neurol Surg. 2006; 34(8):819-24.

23. Café MEM, Rodrigues RC, Viggiano AM. Você conhece esta síndrome?. An Bras Dermatol. 2008; 83(2):167-9.

24. Pascual-Castroviejo I, Pascual-Pascual SI, Velazquez-Fraqua $R$, Viaño J. Sturge-Weber syndrome: study of 55 patients. Can J Neurol Sci. 2008; 35(3):301-7.

25. Petit $F$, Auvin $S$, Lamblin MD, Vallée L. [Myoclonic astatic seizures in a child with SturgeWeber syndrome]. Rev Neurol (Paris). 2008; 164(11):953-6.

26. Delvi MB, Takrouri MS. Anesthesia for encephalo-trigeminal angiomatosis (Sturge-Weber syndrome). Middle East J Anesthesiol. 2006 Feb; 18(4):785-90.

27. Roach ES. Neurocutaneous syndromes. Pediatr Clin North Am. 1992; 39(4):591-620.

28. Bebin EM, Gomez MR. Prognosis in SturgeWeber disease: comparison of unihemispheric and bihemispheric involvement. J Child Neurol. 1988; 3(3):181-4.

RECEBIDO EM: 11/12/2008

ACEITO EM: 13/09/2009

Endereço para correspondência:

Sara Virgínia Paiva Santos

Rua Cidália Menezes, BI 108 ap. 202

Salvador - BA

CEP: $41180-400$

E-mail: svpsfono@yahoo.com.br / svpsfono@gmail.com
29. Moritani T, Kim J, Sato Y, Bonthius D, Smoker WR. Abnormal hypermyelination in a neonate with Sturge-Weber syndrome demonstrated on diffusion-tensor imaging. J Magn Reson Imaging. 2008; 27(3):617-20.

30. Sivaswamy L, Rajamani K, Juhasz C, Maqbool M, Makki M, Chugani HT. The corticospinal tract in Sturge-Weber syndrome: a diffusion tensor tractography study. Brain Dev. 2008; 30(7):447-53. 31. Comi AM. Advances in Sturge-Weber syndrome. Curr Opin Neurol. 2006 Apr; 19(2):124-8.

32. Frankenburg WK, Dodds JB. The Denver developmental screening test. J Pediatr 1967; 71(2):181-91.

33. Marchesan IQ. Avaliação das funções miofuncionais orais. In: Lopes Filho O. Tratado de fonoaudiologia. São Paulo: Tecmed; 2005. p. 727-33.

34. Garcia R, Rossi NF, Giacheti CM. Perfil de habilidades de comunicação de dois irmãos com a Síndrome Alcoólica Fetal. Rev. CEFAC. 2007; 9(4):461-8. dx.doi.org/10.1590/ S1516-18462007000400005

35. Fernandes FDM. Pragmática (Parte D). In: Andrade CRF, Befi-Lopes DM, Fernandes FDM, Wertzner HF. ABFW: Teste de linguagem infantil nas áreas de fonologia, vocabulário, fluência e pragmática. Barueri: Pró-Fono; 2004. p. 83-97.

36. Santos RS, Araújo AP, Porto MA. Early diagnosis of abnormal development of preterm newborns: assessment instruments. J Pediatr. 2008; 84(4):289-99.

37. Freitas LO, Silva TT, Oliveira RT. Manual Denver II. In: Frankenburg WK, et al. Denver II screening manual. Denver, CO: Denver Developmental Materiais. Inc.; 1990.

38. Furkim AM, Silva RG. Conceitos e implicações para prática clínica e para classificação da disfagia orofaríngea neurogênica [CD-Book]. In: Furkim AM, Silva RG. Programas de reabilitação em disfagia neurogênica. São Paulo: Frôntis Editorial; 1999. p. 1-15.

39. Alves NSG. O fundamental da avaliação fonoaudiológica do paciente disfágico. In: Costa M, Castro LP. Tópicos em deglutição e disfagia. São Paulo: Medsi; 2004. p. 9-18. 